\title{
The $\chi_{-y}$-genera of relative Hilbert schemes for linear systems on Abelian and K3 surfaces
}

\author{
Lothar Göttsche and Vivek Shende
}

\begin{abstract}
For an ample line bundle on an Abelian or K3 surface, minimal with respect to the polarization, the relative Hilbert scheme of points on the complete linear system is known to be smooth. We give an explicit expression in quasi-Jacobi forms for the $\chi_{-y}$ genus of the restriction of the Hilbert scheme to a general linear subsystem. This generalizes a result of Yoshioka and Kawai for the complete linear system on the K3 surface, a result of Maulik, Pandharipande, and Thomas on the Euler characteristics of linear subsystems on the K3 surface, and a conjecture of the authors.
\end{abstract}

\section{Introduction}

Let $S$ be a smooth complex algebraic surface, and let $L$ be a line bundle over $S$. Consider a linear system $\mathbb{P}^{\delta} \subset|L|$. Let $\mathcal{C} \rightarrow \mathbb{P}^{\delta}$ be the universal family of curves over the linear system, and let $\mathcal{C}^{[n]}$ be the relative Hilbert schemes of points on the fibres. Under suitable hypotheses, the Euler numbers of the $\mathcal{C}^{[n]}$ control the number of $\delta$-nodal curves in $\mathbb{P}^{\delta}$ [KST11, KS13]. In these cases, the relative Hilbert schemes can be identified with the surface variant of the spaces of stable pairs of Pandharipande and Thomas [PT09, PT10, KT14a, KT14b]. When the spaces $\mathcal{C}^{[n]}$ are smooth, their Euler numbers may be computed by integrating Chern classes. Writing these as "tautological" integrals over $S^{[n]}$ allows the fact (proved in [EGL01]) that all such integrals are determined by the Chern classes of $S$ and $L$ to be imported into enumerative geometry; one concludes that the number of $\delta$-nodal curves in a $\delta$-dimensional slice of a $\delta$-very-ample linear system is given by a universal formula. This result had been previously conjectured by the first author in [Göt98] and previously proven by other methods in [Tze12].

When $S$ is a K3 surface, explicit formulas for the $\chi\left(\mathcal{C}^{[n]}\right)$ are known. The derivation of these is however rather indirect: one shows an equivalence between the stable pairs and Gromov-Witten theories ([MPT10], according to which this is in the spirit of, but does not directly follow from the conjectural threefold equivalence of [MNOP06]) and calculates the latter [BL00]. Similar methods may be expected to work for the Abelian surface; alternatively, the formula for the K3 surface determines the formula for the Abelian surface "by universality".

In [GS14], we replace the topological Euler characteristic $\chi$ with the Hirzebruch $\chi_{-y}$ genus $\left(\chi=\chi_{-1}\right)$. Imitating the formulas of [KST11] leads to putative refined curve counts which conjecturally are given by a universal formula in the Chern numbers. The refinement recovers at

Received 18 July 2013, accepted in final form 5 December 2014.

2010 Mathematics Subject Classification 14C05, 14D20, 14N10, 14 N35.

Keywords: Hilbert schemes of points, Abelian surface, enumerative geometry.

This journal is (C) Foundation Compositio Mathematica 2015. This article is distributed with Open Access under the terms of the Creative Commons Attribution Non-Commercial License, which permits non-commercial reuse, distribution, and reproduction in any medium, provided that the original work is properly cited. For commercial re-use, please contact the Foundation Compositio Mathematica. 


\section{Lothar Göttsche And ViveK Shende}

$y=1$ the counts of complex curves, and conjecturally for a toric surface computes tropical refined Severi degrees [BG14, IM13]. The tropical refined Severi degrees are defined combinatorially, but carry two meaningful enumerative specializations: at $y=1$ they count complex curves, and at $y=-1$ they count real curves [BG14, Mik05].

Moreover, we conjectured in [GS14] an explicit formula for the refined invariants in the case of K3 or Abelian surfaces. Our goal here is to give a derivation of this formula, which in turn determines two of the four series involved in the (still conjectural) formula for a general surface.

The quantity

$$
X_{-y}(x):=\frac{x\left(1-y e^{x\left(y^{1 / 2}-y^{-1 / 2}\right)}\right)}{y^{1 / 2}\left(1-e^{x\left(y^{1 / 2}-y^{-1 / 2}\right)}\right)}
$$

is the normalized power series that defines the genus $\bar{\chi}_{-y}(M)=y^{-\operatorname{dim}(M) / 2} \chi_{-y}(M)$. That is, for a vector bundle $E$ with Chern roots $e_{1}, \ldots, e_{n}$, we define $X_{-y}(E)=\prod_{i=1}^{n} X_{-y}\left(e_{i}\right)$. For a smooth projective variety $M$, we write $X_{-y}(M):=X_{-y}\left(T_{M}\right)$, and by the Riemann-Roch formula [Hir95] we have

$$
\bar{\chi}_{-y}(M):=\int_{M} X_{-y}(M)=y^{-\operatorname{dim} M / 2} \sum_{p, q}(-1)^{p+q} y^{q} \mathrm{~h}^{p, q}(M) .
$$

We collect the $\chi_{-y}$ genera of relative Hilbert schemes over complete linear systems on the Abelian and K3 surfaces into generating series.

Definition 1.1. Throughout we write $L_{g}$ to indicate a line bundle with no higher cohomology whose sections have arithmetic genus $g$. Note for all $g \geqslant 2$ there is an Abelian surface $A_{g}$ carrying a line bundle $L_{g}$ such that the relative Hilbert schemes $\mathcal{C}_{g}^{[n]} \rightarrow\left|L_{g}\right|$ are smooth. We define

$$
\mathbb{A}:=\sum_{g \geqslant 2} \sum_{n \geqslant 0} \bar{\chi}_{-y}\left(\mathcal{C}_{g}^{[n]}\right) t^{n+1-g} q^{g-1} .
$$

Similarly, let $L_{g}$ be a linear system of genus $g$ curves on a K3 surface $K_{g}$ such that the relative Hilbert schemes $\mathcal{C}_{g}^{[n]} \rightarrow\left|L_{g}\right|$ are smooth. We define

$$
\mathbb{K}:=\sum_{g \geqslant 0} \sum_{n \geqslant 0} \bar{\chi}_{-y}\left(\mathcal{C}_{g}^{[n]}\right) t^{n+1-g} q^{g-1} .
$$

We require two more generating series which contain the same information as $\mathbb{A}$. Writing $D=q d / d q$, we define

$$
\begin{aligned}
& \mathbb{H}:=D^{-1} \mathbb{A}=\sum_{g \geqslant 2} \sum_{n \geqslant 0} \bar{\chi}_{-y}\left(\mathcal{C}_{g}^{[n]}\right) t^{n+1-g} \frac{q^{g-1}}{g-1}, \\
& \mathbb{X}:=\frac{\mathbb{H}}{X_{-y}(\mathbb{H})}=\frac{y^{1 / 2}\left(1-e^{\left(y^{1 / 2}-y^{-1 / 2}\right) \mathbb{H}}\right)}{1-y e^{\left(y^{1 / 2}-y^{-1 / 2}\right) \mathbb{H}}} .
\end{aligned}
$$

As we recall in Section 2, when the surface $S$, line bundle $L$, and linear system $\mathbb{P}^{\delta} \subset|L|$ are such that $L$ has no higher cohomology and the relative Hilbert scheme $\mathcal{C}^{[n]} \rightarrow \mathbb{P}^{\delta}$ has nonsingular total space, the Hirzebruch genus $\bar{\chi}_{y}\left(\mathcal{C}^{[n]}\right)$ is given by some universal expression (depending on $n, \delta)$ in the Chern classes of $S, L$. Thus we may write $\bar{\chi}_{-y}\left(\mathcal{C}_{[S, L], \delta}^{[n]}\right)$ for the evaluation of this expression for any $S, L$, or indeed any specification of the Chern numbers $c_{1}(S)^{2}, c_{1}(S) \cdot L$, $L^{2}, c_{2}(S)$. We write $\bar{\chi}_{-y}\left(\mathcal{C}_{[S, L]}^{[n]}\right):=\bar{\chi}_{-y}\left(\mathcal{C}_{[S, L], \chi(L)-1}^{[n]}\right)$ for the evaluation corresponding to the complete linear system. In speaking of $\chi(L), \chi\left(\mathcal{O}_{S}\right), g(L)$, etc., we mean the evaluation on the 


\section{$\chi_{y}$-Genera of RElative HiLberT SCHEMES}

specified Chern numbers of the formulas which usually give these quantities. More generally, in the same way we may 'integrate tautological classes over $\mathcal{C}_{[S, L], \delta}^{[n]}$ ' Arguments similar to those of [Göt98, EGL01, KST11, GS14] establish the following result.

Theorem 1.2. There exist two more series $\mathbb{B}_{1}, \mathbb{B}_{2} \in \mathbb{Q}\left[y^{ \pm 1 / 2}, t^{-1}\right][[t, q]]$ such that the following hold:

$$
\begin{aligned}
& \mathbb{X}^{k} \mathbb{B}_{1}^{K_{S}^{2}} \mathbb{B}_{2}^{L K_{S}} \mathbb{K}^{\chi\left(\mathcal{O}_{S}\right) / 2} \mathbb{A}^{1-\chi\left(\mathcal{O}_{S}\right) / 2}=\sum_{g} \sum_{n \geqslant 0} t^{n-g+1} q^{g-1} \bar{\chi}_{-y}\left(\mathcal{C}_{[S, L], \chi(L)-1-k}^{[n]}\right), \\
& \mathbb{H}^{k} \mathbb{B}_{1}^{K_{S}^{2}} \mathbb{B}_{2}^{L K_{S}} \mathbb{K}^{\chi\left(\mathcal{O}_{S}\right) / 2} \mathbb{A}^{1-\chi\left(\mathcal{O}_{S}\right) / 2}=\sum_{g} \sum_{n \geqslant 0} t^{n-g+1} q^{g-1}\left(\int_{\mathcal{C}_{[S, L]}^{[n]}} X_{-y}\left(\mathcal{C}_{[S, L]}^{[n]}\right) \cap H^{k}\right)
\end{aligned}
$$

The meaning of the sum is that we fix $c_{1}(S)^{2}, c_{2}(S), c_{1}(S) \cdot L$, and vary only $L^{2}$, which we track by $g=g(L)$.

In both formulas, the summand on the right-hand side vanishes unless $g \geqslant k+2+L K_{S}-\chi\left(\mathcal{O}_{S}\right)$. Indeed, this may be checked when $L$ is an actual line bundle with no higher cohomology on an actual surface $S$, where it amounts to $\operatorname{dim}|L| \geqslant k$.

The Hodge polynomials of the relative Hilbert schemes on K3 surfaces were computed by Kawai and Yoshioka; specializing these gives an explicit formula for $\mathbb{K}$. In the present note we will compute $\mathbb{A}$.

We introduce some notation in order to state the answer. Let $z$ be a complex variable and $\tau$ a variable from the complex upper half plane. We write $y=e^{z}$ and $q:=e^{2 \pi i \tau}$. We denote one of the standard theta functions by

$$
\begin{aligned}
\theta(z)=\theta(z, \tau) & :=\sum_{n \in \mathbb{Z}}(-1)^{n} q^{\frac{1}{2}\left(n+\frac{1}{2}\right)^{2}} y^{n+\frac{1}{2}} \\
& =q^{1 / 8}\left(y^{1 / 2}-y^{-1 / 2}\right) \prod_{n>0}\left(1-q^{n}\right)\left(1-q^{n} y\right)\left(1-q^{n} / y\right),
\end{aligned}
$$

and the Eisenstein series of weight 2 by

$$
G_{2}(\tau):=-\frac{1}{24}+\sum_{n>0}\left(\sum_{d \mid n} d\right) q^{n} .
$$

By abuse of notation we also write $\theta(y):=\theta(z), G_{2}(q):=G_{2}(\tau)$. Let ' denote $\partial / \partial z=y \partial / \partial y$, and let $D=(1 / 2 \pi i) \partial / \partial \tau=q d / d q$.

Definition 1.3. We write

$$
A(y, q):=\sum_{n d>0} \operatorname{sgn}(d) n^{2} y^{d} q^{n d}
$$

Here, as also a number of times below, $\sum_{n d>0}$ denotes the sum over pairs $n, d$ of integers with $n d>0$. Thus $A(y, q)$ can be viewed as a theta function for an indefinite lattice, as considered, for example, in [Zag91, GZ98].

Remark 1.4. The sum $A(y, q)$ can be rewritten as follows:

$$
A(y, q)=-\frac{1}{3} \frac{\theta^{\prime \prime \prime}(y)}{\theta(y)}-2 G_{2}(q) \frac{\theta^{\prime}(y)}{\theta(y)}=\frac{1}{\theta(y)}\left(-\frac{1}{6} D \theta^{\prime}(y)-2 G_{2}(q) \theta^{\prime}(y)\right) .
$$




\section{Lothar Göttsche And Vivek Shende}

Proof. The second equality holds by the heat equation $\theta^{\prime \prime}(y)=\frac{1}{2} D \theta(y)$. Let us prove the first equality. Write $y_{1}=e^{z_{1}}$ and $y=e^{z}$ for complex variables $z_{1}$ and $z$. In [Zag91, p. 456, compare (iii) and (vii)] it is proved that

$$
\frac{\theta^{\prime}(0) \theta\left(y_{1} y\right)}{\theta\left(y_{1}\right) \theta(y)}=\frac{y_{1} y-1}{\left(y_{1}-1\right)(y-1)}-\sum_{n d>0} \operatorname{sgn}(d) y_{1}^{n} y^{d} q^{n d}
$$

We take the coefficient of $z_{1}^{2}$ of both sides of (1.1). By the identity [Zag91, Equation (7)] we have

$$
\frac{z_{1} \theta^{\prime}(0)}{\theta\left(y_{1}\right)}=\exp \left(2 \sum_{k \geqslant 2} G_{k}(\tau) \frac{z_{1}^{k}}{k !}\right) \text {. }
$$

This gives

$$
\operatorname{Coeff}_{z_{1}^{2}}\left[\frac{\theta^{\prime}(0) \theta\left(y_{1} y\right)}{\theta\left(y_{1}\right) \theta(y)}\right]=\operatorname{Coeff}_{z_{1}^{3}}\left[\frac{\theta\left(y_{1} y\right)}{\theta(y)}\right]+G_{2}(\tau) \operatorname{Coeff}_{z_{1}}\left[\frac{\theta\left(y_{1} y\right)}{\theta(y)}\right]=\frac{1}{6} \frac{\theta^{\prime \prime \prime}(y)}{\theta(y)}+G_{2}(\tau) \frac{\theta^{\prime}(y)}{\theta(y)} .
$$

On the other hand,

$$
\operatorname{Coeff}_{z_{1}^{2}}\left[\frac{y_{1} y-1}{\left(y_{1}-1\right)(y-1)}-\sum_{n d>0} \operatorname{sgn}(d) y_{1}^{n} y^{d} q^{n d}\right]=-\frac{1}{2} \sum_{n d>0} \operatorname{sgn}(d) n^{2} y^{d} q^{n d}=-\frac{1}{2} A(y, d) .
$$

This proves the claim.

We use the following abbreviation:

$$
[n]_{y}:=\frac{y^{n / 2}-y^{-n / 2}}{y^{1 / 2}-y^{-1 / 2}}=y^{(n-1) / 2}+y^{(n-3) / 2}+\cdots+y^{-(n-1) / 2} .
$$

Theorem 1.5. Let $L_{g}$ be a linear system of genus $g$ curves on an Abelian surface $A_{g}$ such that the relative Hilbert schemes $\mathcal{C}_{g}^{[n]} \rightarrow\left|L_{g}\right|$ are smooth. Then,

$$
\begin{gathered}
\mathbb{A}:=\sum_{g \geqslant 2} \sum_{n \geqslant 0} \bar{\chi}_{-y}\left(\mathcal{C}_{g}^{[n]}\right) t^{n+1-g} q^{g-1}=\left(t+t^{-1}-y^{1 / 2}-y^{-1 / 2}\right) \sum_{n>0, d>0} n^{2}[d]_{y}[d]_{t y^{1 / 2}}[d]_{t y^{-1 / 2}} q^{n d} \\
=\sum_{n>0, d>0} n^{2} \frac{y^{d / 2}-y^{-d / 2}}{y^{1 / 2}-y^{-1 / 2}}\left(t^{d}+t^{-d}-y^{d / 2}-y^{-d / 2}\right) q^{n d} \\
=\frac{1}{y^{1 / 2}-y^{-1 / 2}}\left(A\left(y^{1 / 2} t, q\right)+A\left(y^{1 / 2} / t, q\right)-A(y, q)\right) .
\end{gathered}
$$

The following is a specialization (and slight reformulation) of a result of Kawai and Yoshioka [KY00].

Theorem 1.6 ([KY00]). Let $L_{g}$ be a linear system of genus $g$ curves on a $K 3$ surface $K_{g}$ such that the relative Hilbert schemes $\mathcal{C}_{g}^{[n]} \rightarrow\left|L_{g}\right|$ are smooth. Then

$$
\mathbb{K}:=\sum_{g \geqslant 0} \sum_{n \geqslant 0} \bar{\chi}_{-y}\left(\mathcal{C}_{g}^{[n]}\right) t^{n+1-g} q^{g-1}=\frac{y^{-1 / 2}-y^{1 / 2}}{\Delta(q)} \frac{\theta^{\prime}(0)^{3}}{\theta\left(y^{1 / 2} / t\right) \theta\left(t y^{1 / 2}\right) \theta(y)} .
$$

Finally, we write explicitly the specialization $(y=1)$ to Euler numbers. We denote $\bar{B}_{1}(q, t):=$ $\mathbb{B}_{1}(q, 1, t)$ and $\bar{B}_{2}(q, t):=\mathbb{B}_{2}(q, 1, t)$. In [GS14], we have introduced the function

$$
\widetilde{D G_{2}}(y, q):=\sum_{n, d>0} n[d]_{y}^{2} q^{n d}=\frac{D \log \left(\theta^{\prime}(0) / \theta(y)\right)}{y-2+y^{-1}}
$$




\section{$\chi_{y}$-Genera of Relative Hilbert schemes}

where the second identity is elementary. From the third line in Theorem 1.5, we have

$$
\begin{aligned}
& \mathbb{A}(q, 1, t)=\left(t-2+t^{-1}\right) \widetilde{D G_{2}}(t, q)=D D \log \left(\theta^{\prime}(0) / \theta(t)\right), \\
& \mathbb{H}(q, 1, t)=\left(t-2+t^{-1}\right) \widetilde{D G_{2}}(t, q)=D \log \left(\theta^{\prime}(0) / \theta(t)\right) .
\end{aligned}
$$

From Theorem 1.6 it is easy to see that

$$
\mathbb{K}(q, 1, t)=\frac{\theta^{\prime}(0)^{2}}{\Delta(q) \theta(t)^{2}}=\frac{1}{\left(t-2+t^{-1}\right) q \prod_{n>0}\left(1-q^{n}\right)^{20}\left(1-q^{n} t\right)^{2}\left(1-y^{n} / t\right)^{2}}=\frac{1}{\phi_{10,1}(t, q)},
$$

where, up to normalization, $\phi_{10,1}(t, q)$ is the unique Jacobi cusp form on $\operatorname{SL}(2, \mathbb{Z})$ of weight 10 and index 1 . It is easy to see that $X_{-1}(x)=(1+x)$, thus

$$
\mathbb{X}(q, 1, t)=\frac{D \log \left(\theta^{\prime}(0) / \theta(t)\right)}{1+D \log \left(\theta^{\prime}(0) / \theta(t)\right)} .
$$

Putting this together, we get the following result.

COROLlary 1.7. The generating series of integrals against the hyperplane class is

$$
\begin{aligned}
\sum_{g \geqslant k+2+L K_{S}-\chi\left(\mathcal{O}_{S}\right)} & \sum_{n \geqslant 0}\left(\int_{\mathcal{C}_{[S, L]}^{[n]}} c\left(\mathcal{C}_{[S, L]}^{[n]}\right) \cap H^{k}\right) t^{n-g+1} q^{g-1} \\
= & \left(D \log \frac{\theta^{\prime}(0)}{\theta(t)}\right)^{k} \bar{B}_{1}^{K_{S}^{2}} \bar{B}_{2}^{L K_{S}}\left(\frac{\theta^{\prime}(0)^{2}}{\Delta(q) \theta(t)^{2}}\right)^{\chi\left(\mathcal{O}_{S}\right) / 2}\left(D D \log \frac{\theta^{\prime}(0)}{\theta(t)}\right)^{1-\chi\left(\mathcal{O}_{S}\right) / 2} .
\end{aligned}
$$

The generating series of Euler characteristics is

$$
\begin{aligned}
& \sum_{g \geqslant k+2+L K_{S}-\chi\left(\mathcal{O}_{S}\right)} \sum_{n \geqslant 0} \chi\left(\mathcal{C}_{[S, L], \chi(L)-1-k}^{[n]}\right) t^{n-g+1} q^{g-1} \\
& =\left(\frac{D \log \left(\theta^{\prime}(0) / \theta(t)\right)}{1+D \log \left(\theta^{\prime}(0) / \theta(t)\right)}\right)^{k} \bar{B}_{1}^{K_{S}^{2}} \bar{B}_{2}^{L K_{S}}\left(\frac{\theta^{\prime}(0)^{2}}{\Delta(q) \theta(t)^{2}}\right)^{\chi\left(\mathcal{O}_{S}\right) / 2}\left(D D \log \frac{\theta^{\prime}(0)}{\theta(t)}\right)^{1-\chi\left(\mathcal{O}_{S}\right) / 2} .
\end{aligned}
$$

As $D \log \frac{\theta^{\prime}(0)}{\theta(t)} \in q \mathbb{Q}\left[t^{ \pm 1}\right][[q]]$, we can develop

$$
\left(\frac{D \log \left(\theta^{\prime}(0) / \theta(t)\right)}{1+D \log \left(\theta^{\prime}(0) / \theta(t)\right)}\right)^{k}=\sum_{m \geqslant 0}(-1)^{m}\left(\begin{array}{c}
m+k-1 \\
k-1
\end{array}\right)\left(D \log \frac{\theta^{\prime}(0)}{\theta(t)}\right)^{m+k}
$$

thus for K3 surfaces the result specializes to formula (7) and Theorem 6 of [MPT10].

We return to the setting of [GS14], where polynomials $N_{[S, L], \delta}^{i}(y)$ (called $\bar{N}_{[S, L], \delta}^{i}(y)$ there) were defined by the following formula, in which $g=g(L)$ :

$$
\sum_{n \geqslant 0} \bar{\chi}_{-y}\left(\mathcal{C}_{[S, L], \delta}^{[n]}\right) t^{n+1-g}=\sum_{i=0}^{\infty} N_{[S, L], \delta}^{i}(y)\left(t+t^{-1}-y^{1 / 2}-y^{-1 / 2}\right)^{g-i-1} .
$$

This formula refines the change of variable used to pass from Euler numbers of Hilbert schemes to enumerative information (of the sort sometimes called Gopakumar-Vafa or 'BPS' invariants). In the good situation where $[S, L]$ comes from a line bundle on a surface with no higher cohomology and the appropriate relative Hilbert schemes are nonsingular, $\left.N^{\delta}\right|_{y=1}$ counts the number of $\delta$ nodal curves in a general $\mathbb{P}^{\delta} \subset|L|$, by [KST11].

According to [GS14, Conjecture 40], we expect the vanishing $N_{[S, L], \delta}^{i}=0$ for $i>\delta$. This was proven in case $K_{S}$ is numerically trivial. Moreover, in this case, or assuming the vanishing 


\section{Lothar Göttsche And Vivek Shende}

conjecture and in terms of two undetermined power series, [GS14, Conjecture 67] gives a conjectural generating function for the highest-order term $N_{[S, L], \delta}^{\delta}$. To establish this formula, and to better understand the $N^{i}$, it remains to develop the series introduced here in the variable $x=\left(t+t^{-1}-y^{1 / 2}-y^{-1 / 2}\right)$.

From Theorem 1.2 and (1.2), we obtain the following generating series.

Corollary 1.8. Let $S, L$ be arbitrary and let $g$ be the arithmetic genus of $L$; then

$$
\sum_{i} N_{[S, L], \chi(L)-1-k}^{i}(y) x^{g-i-1}=\operatorname{Coeff}_{q^{g-1}}\left[\mathbb{X}^{k} \mathbb{B}_{1}^{K_{S}^{2}} \mathbb{B}_{2}^{L K_{S}} \mathbb{K}^{\chi\left(\mathcal{O}_{S}\right) / 2} \mathbb{A}^{1-\chi\left(\mathcal{O}_{S}\right) / 2}\right] .
$$

We set

$$
\widetilde{\Delta}(y, q):=\frac{\Delta(q) \theta(y)^{2}}{\left(y-2+y^{-1}\right) \theta^{\prime}(0)^{2}}=q \prod_{n>0}\left(1-q^{n}\right)^{20}\left(1-q^{n} y\right)^{2}\left(1-q^{n} / y\right)^{2} .
$$

Corollary 1.9 ([GS14, Conjecture 68]). If $K_{S}$ is numerically trivial, then

$$
N_{[S, L], \chi(L)-1-k}^{\chi(L)-1-k}(y)=\operatorname{Coeff}_{q^{g-1}} \frac{\widetilde{D G}_{2}(y, q)^{k}\left(D \widetilde{D G}_{2}(y, q)\right)^{1-\chi\left(\mathcal{O}_{S}\right) / 2}}{\widetilde{\Delta}(y, q)^{\chi\left(\mathcal{O}_{S}\right) / 2}} .
$$

More generally, we want expressions for all the $N^{i}$, or in other words, we want to expand $\mathbb{A}$ and $\mathbb{K}$ in $x$ rather than $t$.

We define polynomials $s_{n}(y)$ and their generating function $S(y, x)$ by

$$
\begin{aligned}
s_{n}(y) & :=\sum_{k=0}^{n}\left(\begin{array}{l}
n \\
k
\end{array}\right)^{2} y^{k-n / 2}=\operatorname{Coeff}_{t^{n}}\left(y^{-1 / 2}(1+t)(1+t y)\right)^{n}, \\
S(y, x) & :=\sum_{n \geqslant 0}(-1)^{n} \frac{s_{n}(y)}{\left(y^{1 / 2}-y^{-1 / 2}\right)^{2 n+1}} \frac{x^{n+1}}{n+1} .
\end{aligned}
$$

Then we have the following equalities.

THEOREM 1.10 .

$$
\begin{aligned}
\mathbb{K} & =\frac{1}{\widetilde{\Delta}(y, q)}\left(\frac{1}{x}+\frac{1}{y^{1 / 2}-y^{-1 / 2}} \sum_{n d>0} \operatorname{sgn}(d) e^{S(y, x)(d-n)} y^{d} q^{n d}\right), \\
\mathbb{A} & =\frac{1}{y^{1 / 2}-y^{-1 / 2}}\left(\sum_{n d>0} \operatorname{sgn}(d)\left(e^{S(y, x) d}-1\right) n^{2}\left(y^{d}-1\right) q^{n d}\right), \\
\mathbb{H} & =\frac{1}{y^{1 / 2}-y^{-1 / 2}}\left(\sum_{n d>0} \operatorname{sgn}(d)\left(e^{S(y, x) d}-1\right) \frac{n}{d}\left(y^{d}-1\right) q^{n d}\right) .
\end{aligned}
$$

To see explicitly the development of $\mathbb{A}$ and $\mathbb{K}$ in $x$, we expand

$$
e^{S(y, x) z}=: \sum_{n \geqslant 0} P_{n}(y, z) \frac{x^{n}}{\left(y^{1 / 2}-y^{-1 / 2}\right)^{n}}
$$

with $P_{n}(y, z) \in \mathbb{Q}[1 /(y-1), y, z]$, for example,

$$
\begin{gathered}
P_{0}=1, \quad P_{1}=z, \quad P_{2}=\frac{z^{2}}{2}-\frac{z}{2} \frac{y+1}{y-1}, \quad P_{3}=\frac{z^{3}}{6}-\frac{z^{2}}{2} \frac{y+1}{y-1}+\frac{z}{3} \frac{y^{2}+4 y+1}{(y-1)^{2}}, \\
P_{4}=\frac{z^{4}}{24}-\frac{z^{3}}{4} \frac{y+1}{y-1}+\frac{z^{2}}{24} \frac{11 y^{2}+38 y+11}{(y-1)^{2}}-\frac{z}{4} \frac{y^{3}+9 y^{2}+9 y+1}{(y-1)^{3}} .
\end{gathered}
$$




\section{$\chi_{y}$-Genera of RElative HiLberT SCHEMES}

Remark 1.11. It is remarkable that the generating functions for Abelian and K3 surfaces are determined by the same polynomials $P_{i}$ : Using Corollary 1.8, we have on a K3 surface

$$
\sum_{g} N_{\left[S, L_{g}\right]}^{g}(y) q^{g-1}=\frac{1}{\widetilde{\Delta}(y, q)}
$$

and for $h \geqslant 1$,

$$
\sum_{g} N_{\left[S, L_{g}\right]}^{g-h}(y) q^{g-1}=\frac{1}{\widetilde{\Delta}(y, q)} \sum_{n d>0} \operatorname{sgn}(d) \frac{P_{h-1}(y, d-n)}{\left(y^{1 / 2}-y^{-1 / 2}\right)^{h}} y^{d} q^{n d} .
$$

On an Abelian surface, we have for $h \geqslant 2$,

$$
\sum_{g} N_{\left[A, L_{g}\right]}^{g-h}(y) q^{g-1}=\sum_{n d>0} \operatorname{sgn}(d) \frac{P_{h-1}(y, d)}{\left(y^{1 / 2}-y^{-1 / 2}\right)^{h}} n^{2}\left(y^{d}-1\right) q^{n d} .
$$

In fact, we first arrived at the formula asserted in Theorem 1.5 in the following manner. The first author conjectured, on the basis of numerical evidence, that (1.4) and (1.5) held for some undetermined coefficients $P_{i}$. This suffices in principle to (conjecturally) determine $\mathbb{A}$ from $\mathbb{K}$. Zagier made this determination explicit, providing a formula for the $P_{i}$ and for $\mathbb{A}$. Finally, we have reversed the procedure, proving the formula for $\mathbb{A}$ geometrically and deriving (1.4) and (1.5) as consequences.

\section{Universality arguments}

In this section we give the proof of Theorem 1.2.

Definition 2.1. Let $S$ be a surface, let $L$ be a line bundle on $S$, and let $L^{[n]}$ be the corresponding tautological vector bundle on $S^{[n]}$. Let $e^{x}$ denote a trivial line bundle with nontrivial $\mathbb{C}^{*}$-action with equivariant first Chern class $x$. Then we define

$$
D^{S, L}(x, y, t):=\sum_{n \geqslant 0} t^{n} \int_{S^{[n]}} X_{-y}\left(T S^{[n]}\right) \frac{c_{n}\left(L^{[n]} \otimes e^{x}\right)}{X_{-y}\left(L^{[n]} \otimes e^{x}\right)}
$$

(note that this differs from [GS14] by the normalization by $y^{-\operatorname{dim} / 2}$ ).

As explained in [GS14, Proposition 47], for a linear subsystem $\mathbb{P}^{\delta} \subset|L|$ such that the relative Hilbert schemes $\mathcal{C}_{[S, L], \delta}^{[n]} \rightarrow \mathbb{P}^{\delta}$ are all smooth - for example, a general $\delta$-dimensional linear subsystem when $L$ is $\delta$-very-ample [KST11] - we may extract the $\bar{\chi}_{-y}$ genera by taking a residue:

$$
\sum_{n \geqslant 0} \bar{\chi}_{-y}\left(\mathcal{C}_{[S, L], \delta}^{[n]}\right) t^{n}=\operatorname{res}_{x=0}\left[D^{S, L}(x, y, t)\left(\frac{X_{-y}(x)}{x}\right)^{\delta+1}\right] d x .
$$

Since $D^{S, L}$ is defined by a tautological integral, by [EGL01] it depends only on the Chern numbers $c_{2}(S), c_{1}(S)^{2}, c_{1}(S) \cdot c_{1}(L), c_{1}(L)^{2}$. Thus we may make sense of it for arbitrary values of these quantities. Thus we view $(2.1)$ as defining the quantities $\bar{\chi}_{-y}\left(\mathcal{C}_{[S, L], \delta}^{[n]}\right)$ in terms of $\delta, n$, and the Chern numbers of $S$ and $L$, without any assumptions on even the existence of such a surface and line bundle.

The change of variable

$$
q(x)=\frac{x}{X_{-y}(x)}=\frac{y^{1 / 2}\left(1-e^{x\left(y^{1 / 2}-y^{-1 / 2}\right)}\right)}{1-y e^{x\left(y^{1 / 2}-y^{-1 / 2}\right)}}
$$




\section{Lothar Göttsche And ViveK Shende}

is inverse to

$$
x(q)=\frac{\log \left(1-y^{1 / 2} q\right)-\log \left(1-y^{-1 / 2} q\right)}{y^{-1 / 2}-y^{1 / 2}}=\sum_{n>0}[n]_{y} \frac{q^{n}}{n} .
$$

We find

$$
\frac{d x}{d q}=\frac{1}{\left(1-y^{-1 / 2} q\right)\left(1-y^{1 / 2} q\right)} .
$$

Plugging this into the residue formula (2.1), and writing for convenience

$$
\bar{D}^{S, L}(q, y, t):=D^{S, L}(x(q), y, t),
$$

we find

$$
\begin{aligned}
\sum_{n \geqslant 0} \bar{\chi}_{-y}\left(\mathcal{C}_{[S, L], \delta}^{[n]}\right) t^{n} & =\operatorname{res}_{q=0}\left[\bar{D}^{S, L}(q, y, t) q^{-(\delta+1)} \frac{1}{\left(1-y^{-1 / 2} q\right)\left(1-y^{1 / 2} q\right)}\right] \\
& =\operatorname{Coeff}_{q^{\delta}}\left[\bar{D}^{S, L}(q, y, t) \frac{1}{\left(1-y^{-1 / 2} q\right)\left(1-y^{1 / 2} q\right)}\right] .
\end{aligned}
$$

As the term in square brackets is a power series, we may sum to obtain

$$
\sum_{\delta \geqslant 0} \sum_{n \geqslant 0} \bar{\chi}_{-y}\left(\mathcal{C}_{[S, L], \delta}^{[n]}\right) t^{n} q^{\delta}=\frac{\bar{D}^{S, L}(q, y, t)}{\left(1-y^{-1 / 2} q\right)\left(1-y^{1 / 2} q\right)} .
$$

Since $X_{-y}$ is a genus, by [EGL01] there exist power series $a_{0}, a_{1}, a_{2}, a_{3} \in \mathbb{Q}\left[y^{ \pm 1 / 2}\right][[t, x]]$ such that $D^{S, L}(x, y, t)=a_{0}^{\chi(L)} a_{1}^{K_{S}^{2}} a_{2}^{L K_{S}} a_{3}^{\chi\left(\mathcal{O}_{S}\right)}$ (for a detailed argument, see [GS14, Section 3.2]). Setting

$$
A_{i}(q, y, t):=a_{i}(x(q), y, t) \in \mathbb{Q}\left[y^{ \pm 1 / 2}\right][[t, q]]
$$

we get

$$
\sum_{\delta \geqslant 0} \sum_{n \geqslant 0} \bar{\chi}_{-y}\left(\mathcal{C}_{[S, L], \delta}^{[n]}\right) t^{n} q^{\delta}=A_{0}^{\chi(L)} A_{1}^{K_{S}^{2}} A_{2}^{L K_{S}} A_{3}^{\chi\left(\mathcal{O}_{S}\right)} \frac{1}{\left(1-y^{-1 / 2} q\right)\left(1-y^{1 / 2} q\right)}
$$

Note that by $(2.3)$, the coefficient of $q^{0}$ in $\bar{D}^{S, L}(x, y, t)$ is $\left(\left(1-y^{-1 / 2} t\right)\left(1-y^{1 / 2} t\right)\right)^{g-1}$ for $g$ the arithmetic genus of a curve in $|L|$. Thus $A_{i}(q, y, t) \in\left(\left(1-y^{-1 / 2} t\right)\left(1-y^{1 / 2} t\right)\right)^{l_{i}}+q \mathbb{Q}\left[y^{ \pm 1 / 2}\right][[t, q]]$ with $l_{0}=1, l_{1}=0, l_{2}=1, l_{3}=-1$.

If $R$ is a commutative ring, and $f \in R[[q]]$ is an invertible power series, we denote by $f^{-1}$ its compositional inverse. Let

$$
\mathbb{X}(q, y, t):=\left(\frac{q t}{A_{0}}\right)^{-1} \in \mathbb{Q}\left[y^{ \pm 1 / 2}, t^{-1}\right][[t, q]] .
$$

This is set up so that

$$
\frac{\mathbb{X}(q, y, t)}{A_{0}(\mathbb{X}, y, t)}=\frac{q}{t}
$$

and hence $A_{0}(\mathbb{X}, y, t)=q^{-1} t \mathbb{X}(q, y, t)$.

Writing $\mathbb{B}_{1}(q, y, t):=A_{1}(\mathbb{X}, y, t), \mathbb{B}_{2}(q, y, t):=A_{2}(\mathbb{X}, y, t) q / t$, and $\mathbb{B}_{3}(q, y, t):=A_{3}(\mathbb{X}, y, t) t / q$, the substitution $q \mapsto \mathbb{X}$ gives

$$
\sum_{\delta \geqslant 0} \sum_{n \geqslant 0} \bar{\chi}_{-y}\left(\mathcal{C}_{[S, L], \delta}^{[n]}\right) t^{n+1-g_{\mathbb{X}} \delta}=\frac{(\mathbb{X} / q)^{\chi(L)} \mathbb{B}_{1}^{K_{S}^{2}}\left(\mathbb{B}_{2} / q\right)^{L K_{S}}\left(\mathbb{B}_{3} \cdot q\right)^{\chi\left(\mathcal{O}_{S}\right)}}{\left(1-y^{1 / 2} \mathbb{X}\right)\left(1-y^{-1 / 2} \mathbb{X}\right)}
$$




\section{$\chi_{y}$-Genera of RELATive HiLbert schemes}

As in [Göt98], we use the residue formula. Let $R$ be a commutative ring, and let $f \in R[[q]]$ and $g \in a q+q^{2} R[[q]]$, with $a$ invertible in $R$; then

$$
f=\left.\sum_{k=0}^{\infty} g(q)^{k}\left[\frac{f(q) D g(q)}{g(q)^{k+1}}\right]\right|_{q=0} .
$$

We apply this to $(2.5)$ with $g(q)=\mathbb{X}$. On the one hand, $\sum_{n \geqslant 0} \chi_{-y}\left(\mathcal{C}_{[S, L], \delta}^{[n]}\right) t^{n+1-g}$ is the coefficient of $\mathbb{X}^{\delta}$ of the right-hand side. On the other, taking the coefficient by the residue formula above gives, with $g$ again the arithmetic genus of a curve in $|L|$,

$$
\begin{aligned}
\sum_{n \geqslant 0} \bar{\chi}_{-y}\left(\mathcal{C}_{[S, L], \delta}^{[n]}\right) t^{n+1-g} & =\left.\frac{D \mathbb{X} \cdot \mathbb{X}^{-\delta-1} \cdot(\mathbb{X} / q)^{\chi(L)} \mathbb{B}_{1}^{K_{S}^{2}}\left(\mathbb{B}_{2} / q\right)^{L K_{S}}\left(\mathbb{B}_{3} \cdot q\right)^{\chi\left(\mathcal{O}_{S}\right)}}{\left(1-y^{1 / 2} \mathbb{X}\right)\left(1-y^{-1 / 2} \mathbb{X}\right)}\right|_{q=0} \\
& =\operatorname{Coeff}_{q^{g-1}} \frac{D \mathbb{X} \cdot(\mathbb{X})^{\chi(L)-\delta-1} \mathbb{B}_{1}^{K_{S}^{2}} \mathbb{B}_{2}^{L K_{S}} \mathbb{B}_{3}^{\chi\left(\mathcal{O}_{S}\right)}}{\left(1-y^{1 / 2} \mathbb{X}\right)\left(1-y^{-1 / 2} \mathbb{X}\right)}
\end{aligned}
$$

We collect terms with fixed $k=\chi(L)-1-\delta$; that is, (if we assume that $L$ now has higher cohomology) $k$ is the number of point conditions we impose to cut down to $\mathbb{P}^{\delta}$. We now explicitly note the genus of the line bundle appearing in its subscript. Note that we always have $\delta \geqslant 0$, which by definition of $k$ and $\chi(L)=g-L K_{S}-1+\chi\left(\mathcal{O}_{S}\right)$ translates into $g \geqslant k+2+L K_{S}-\chi\left(\mathcal{O}_{S}\right)$.

Corollary 2.2. Fix $k \geqslant 0$; then

$$
\sum_{g \geqslant k+2+L K_{S}-\chi\left(\mathcal{O}_{S}\right)} \sum_{n \geqslant 0} \bar{\chi}_{-y}\left(\mathcal{C}_{\left[S, L_{g}\right], \chi\left(L_{g}\right)-1-k}^{[n]}\right) t^{n-g+1} q^{g-1}=\frac{\mathbb{X}^{k} \mathbb{B}_{1}^{K_{S}^{2}} \mathbb{B}_{2}^{L_{g} K_{S}} \mathbb{B}_{3}^{\chi\left(\mathcal{O}_{S}\right)} D \mathbb{X}}{\left(1-y^{-1 / 2} \mathbb{X}\right)\left(1-y^{1 / 2} \mathbb{X}\right)} .
$$

In particular, when $S=A$ is an Abelian surface,

$$
\frac{D \mathbb{X}}{\left(1-y^{-1 / 2} \mathbb{X}\right)\left(1-y^{1 / 2} \mathbb{X}\right)}=\sum_{g \geqslant 2} \sum_{n \geqslant 0} \bar{\chi}_{-y}\left(\mathcal{C}_{\left[A, L_{g}\right], \chi\left(L_{g}\right)-1}^{[n]}\right) t^{n-g+1} q^{g-1}=: \mathbb{A} .
$$

Note that

$$
\frac{D \mathbb{X}}{\left(1-y^{-1 / 2} \mathbb{X}\right)\left(1-y^{1 / 2} \mathbb{X}\right)}=D\left(\frac{\log \left(1-y^{-1 / 2} \mathbb{X}\right)-\log \left(1-y^{1 / 2} \mathbb{X}\right)}{y^{-1 / 2}-y^{1 / 2}}\right)
$$

Thus, by $\mathbb{H}=D^{-1} \mathbb{A}$, we see

$$
\mathbb{H}=\frac{\log \left(1-y^{1 / 2} \mathbb{X}\right)-\log \left(1-y^{-1 / 2} \mathbb{X}\right)}{y^{-1 / 2}-y^{1 / 2}}=x(\mathbb{X}) .
$$

We have already seen how to invert this function:

$$
\mathbb{X}=q(\mathbb{H})=\frac{\mathbb{H}}{X_{-y}(\mathbb{H})}=\frac{y^{1 / 2}\left(1-e^{\mathbb{H}\left(y^{1 / 2}-y^{-1 / 2}\right)}\right)}{1-y e^{\mathbb{H}\left(y^{1 / 2}-y^{-1 / 2}\right)}}
$$

Similarly, when $S=K$ is a K3 surface,

$$
\mathbb{A B}_{3}^{2}=\sum_{g \geqslant 0} \sum_{n \geqslant 0} \bar{\chi}_{-y}\left(\mathcal{C}_{\left[K, L_{g}\right], \chi\left(L_{g}\right)-1}^{[n]}\right) t^{n-g+1} q^{g-1}=: \mathbb{K},
$$

and so $\mathbb{B}_{3}=(\mathbb{K} / \mathbb{A})^{1 / 2}$. Putting everything together, we obtain a proof of the first formula of Theorem 1.2. 


\section{Lothar Göttsche And Vivek Shende}

We now prove the second formula. The argument takes place for fixed $[S, L]$. Write $H$ for the pullback of the hyperplane class from $|L|$. Let

$$
Z_{[S, L]}(x, y, t):=t^{1-g} D^{S, L}(x, y, t) q(x)^{-\chi(L)} .
$$

Equation (2.1) asserts that when the relevant spaces are smooth, we have

$$
\begin{aligned}
\sum_{n \geqslant 0} \bar{\chi}_{-y}\left(\mathcal{C}_{[S, L], \chi(L)-1-k}^{[n]}\right) t^{n+1-g} & =\operatorname{res}_{x=0}\left[t^{1-g} D^{S, L}(x, y, t) q(x)^{-\delta-1}\right] d x \\
& =\operatorname{res}_{x=0}\left[Z_{[S, L]}(x, y, t) q(x)^{k}\right] d x
\end{aligned}
$$

By the same proof, if the $\mathcal{C}_{[S, L]}^{[n]}$ are smooth, we have

$$
\sum_{n \geqslant 0}\left(\int_{\mathcal{C}_{[S, L]}^{[n]}} X_{-y}\left(\mathcal{C}_{[S, L]}^{[n]}\right) \cap H^{k}\right) t^{n+1-g}=\operatorname{res}_{x=0}\left[Z_{[S, L]}(x, y, t) x^{k}\right] d x .
$$

Write $f(q, y, t):=\mathbb{B}_{1}^{K_{S}^{2}} \mathbb{B}_{2}^{L K_{S}} \mathbb{K}^{\chi\left(\mathcal{O}_{S}\right) / 2} \mathbb{A}^{1-\chi\left(\mathcal{O}_{S}\right) / 2}$. We have shown

$$
\operatorname{res}_{x=0}\left[Z_{[S, L]}(x, y, t) q(x)^{k}\right] d x=\operatorname{Coeff}_{q^{g-1}}\left[\mathbb{X}^{k} f(q, y, t)\right] \text {. }
$$

Let again $x(q)$ from $(2.2)$ be the compositional inverse of $q(x)$. Write $x(q)^{k}:=\sum_{l \geqslant k} a_{l}(y) q^{l}$, so that $\sum_{l \geqslant k} a_{l}(y) q(x)^{l}=x^{k}$. Thus we get

$$
\begin{aligned}
& \operatorname{res}_{x=0}\left[Z_{[S, L]}(x, y, t) x^{k}\right] d x=\operatorname{res}_{x=0}\left[\sum_{l \geqslant k} a_{l}(y) Z_{[S, L]}(x, y, t) q(x)^{l}\right] d x \\
& \quad=\operatorname{Coeff}_{q^{g-1}}\left[\sum_{l \geqslant k} a_{l}(y) \mathbb{X}^{l} f(q, y, t)\right]=\operatorname{Coeff}_{q^{g-1}}\left[x(\mathbb{X})^{k} f(q, y, t)\right]=\operatorname{Coeff}_{q^{g-1}}\left[\mathbb{H}^{k} f(q, y, t)\right] .
\end{aligned}
$$

The last equality is by (2.6).

\section{Calculations for the Abelian surface}

Kawai and Yoshioka determined $\mathbb{K}$ by comparing various moduli spaces of stable sheaves and stable pairs on a K3 surface [KY00]. A modification of their argument suffices to determine $\mathbb{A}$ except for the coefficient of $t^{0}$, and a vanishing result in [GS14] allows us to determine this coefficient from the rest.

\subsection{Yoshioka's lemma}

Lemma 3.1 ([Yos99, Lemma 2.1]). Let $X$ be a smooth projective surface with polarization $H$, and let $C$ be a curve class minimizing $C$.H. For a sheaf $F$ with $c_{1}(F)=d C$, we write $\operatorname{deg}(F)=$ $d=\left(c_{1}(F) \cdot H\right) /(C \cdot H)$. Let $(r, d)$ and $\left(r_{1}, d_{1}\right)$ be pairs of integers such that $r_{1} d-d_{1} r=1$, with $r \geqslant 0$ and $r_{1}>0$. Let $\left(r_{2}, d_{2}\right):=(r, d)-\left(r_{1}, d_{1}\right)$. Let $E_{i}$ be of rank $r_{i}$ and degree $d_{i}$, and let $E_{1}$ always be a vector bundle.

- If $E_{1}$ and $E_{2}$ are $\mu$-stable, then every nontrivial extension

$$
0 \rightarrow E_{1} \rightarrow E \rightarrow E_{2} \rightarrow 0
$$

is $\mu$-stable. 


\section{$\chi_{y}$-Genera of RELATive HiLbert schemes}

- If $E_{1}$ and $E$ are $\mu$-stable, then for any vector subspace $V \subset \operatorname{Hom}\left(E_{1}, E\right)$, if the evaluation map $V \otimes E_{1} \rightarrow E$ is not injective, then it is surjective in codimension 1. Moreover,

$*$ if $V \otimes E_{1} \rightarrow E$ is injective, then the cokernel is $\mu$-stable;

$*$ if $V \otimes E_{1} \rightarrow E$ is surjective in codimension 1, the kernel is $\mu$-stable.

Remark 3.2. In [Yos99], this lemma is proven under the assumption that $N S(X)=\mathbb{Z}$, but in $[\mathrm{KY} 00]$ it is pointed out that the proof only requires the assumption stated above.

Note that if $d=1$, that is, if we are looking at sheaves with $c_{1}=C$, then the condition $r_{1} d-d_{1} r=1$ is always satisfied by $\left(r_{1}, d_{1}\right)=(1,0)$, hence we may always take $E_{1}=\mathcal{O}_{X}$. We now extract explicitly the special cases we will be concerned with.

Corollary 3.3. Let $X$ be a smooth projective surface with polarization $H$, and let $C$ be a curve class minimizing C.H.

(i) Assume that $F$ is $\mu$-stable and $c_{1}(F)=C$. Then every nontrivial extension $0 \rightarrow \mathcal{O}_{X} \rightarrow$ $E \rightarrow F \rightarrow 0$ is $\mu$-stable.

(ii) Assume that $E$ is $\mu$-stable of positive rank and $c_{1}(E)=C$. Then any nonzero section induces an exact sequence $0 \rightarrow \mathcal{O}_{X} \rightarrow E \rightarrow F \rightarrow 0$ and $F$ is $\mu$-stable.

Proof. The only thing which is not immediate from the lemma is to check the possibility in the second case that $\mathcal{O}_{X} \rightarrow E$ is surjective in codimension 1 rather than injective. But then in any case either $E$ must be torsion (which it is not by assumption), or the map from $\mathcal{O}_{X}$ must be an isomorphism in codimension 1 , in which case the kernel must be a torsion subsheaf of $\mathcal{O}_{X}$, hence zero.

Let $\mathcal{M}(r, d, e)$ denote the moduli space of semistable sheaves of rank $r$, degree $d$, and Euler number $e$ (note that we are not indexing by the Mukai vector). From now on, we will assume that $\mathcal{M}(r, d, e)$ consists of only $\mu$-stable sheaves. Let $\mathcal{P}^{1}(r, d, e)$ be the space of "coherent systems" [LP95], that is, it parameterizes a stable sheaf (of rank $r$, degree $d$, and Euler number $e$ ) plus a section, up to isomorphism. This corresponds to a special choice of the stability condition for pairs, which ensures that a pair of sheaf and section is stable if and only if the sheaf is stable. There is a forgetful map $\mathcal{P}^{1}(r, d, e) \rightarrow \mathcal{M}(r, d, e)$ with fibre $\mathbb{P H}^{0}(E)$ over a sheaf $E$.

The corollary above implies the existence of another map:

Corollary 3.4. For $r \geqslant 0$, there exists a morphism $\mathcal{P}^{1}\left(r+1, d, e+\chi\left(\mathcal{O}_{X}\right)\right) \rightarrow \mathcal{M}(r, d, e)$ which takes $\mathcal{O}_{X} \rightarrow E$ to its cokernel. The fibre over a sheaf $F$ is $\mathbb{P E x t}^{1}\left(F, \mathcal{O}_{X}\right)$.

Let us consider the space $\mathcal{P}^{1}(0,1, e)$. This by definition consists of a stable, rank 0 sheaf $E$ together with a section $\mathcal{O}_{X} \rightarrow E$. By stability, $E$ is a pure sheaf supported on a curve (that is, torsion free with rank 1 on its support). As explained in [PT10, Appendix B], dualizing gives an isomorphism between $\mathcal{P}^{1}(0,1, e)$ and the relative Hilbert scheme of degree $e+g-1$, where $g$ is the arithmetic genus of the support of $E$, over the moduli space $\mathcal{M}$ of curves of degree $d C$ on $S$.

\subsection{A relation between moduli spaces}

We now specialize to $K_{X}=\mathcal{O}_{X}$. Note in this case that if $E$ is any stable sheaf with rank 0 or positive first Chern class, then

$$
\mathrm{H}^{2}(E)=\operatorname{Hom}\left(E, K_{X}\right)^{*}=\operatorname{Hom}\left(E, \mathcal{O}_{X}\right)^{*}=0 .
$$




\section{Lothar Göttsche And ViveK Shende}

In the rank 0 case the last equality is obvious; for positive rank it is ensured by stability. Additionally, we have $\operatorname{Ext}^{1}\left(F, \mathcal{O}_{X}\right)=\mathrm{H}^{1}(F)^{*}$ by Serre duality. Thus the dimensions of the fibres of the two maps to $\mathcal{M}(r, d, e)$ are related:

$$
\mathcal{P}^{1}(r, d, e) \stackrel{\mathbb{P H}^{0}(F)}{\longrightarrow} \mathcal{M}(r, d, e) \stackrel{\mathbb{P H} H^{1}(F)}{\longleftarrow} \mathcal{P}^{1}\left(r+1, d, e+\chi\left(\mathcal{O}_{X}\right)\right) .
$$

We indicate throughout the first map by $\mathcal{P} \rightarrow \mathcal{M}$ and the second by $\mathcal{M} \leftarrow \mathcal{P}$.

We denote the Hodge polynomial of $V$ by $[V]$. We write $\mathbb{L}$ for the Hodge polynomial of the affine line, and $[n]=\left[\mathbb{P}^{n-1}\right]$ for $n \in \mathbb{Z}_{>0}$. We also write $[0]=0$ and $[-n]=-\mathbb{L}^{-n}[n]$.

Let $\mathcal{M}(r, d, e)_{s}$ denote the locus with $h^{0}=s$. Since the map $\mathcal{P} \rightarrow \mathcal{M}$ is given on the strata above as the projectivization of a vector bundle, we have

$$
\left[\mathcal{P}^{1}(r, d, e)\right]=\sum_{i}[e+i]\left[\mathcal{M}(r, d, e)_{e+i}\right] .
$$

Considering instead the map $\mathcal{M} \leftarrow \mathcal{P}$ and using the vanishing of $h^{2}(F)$ to write $\chi(F)=h^{0}(F)-$ $h^{1}(F)$, we have

$$
\left[\mathcal{P}^{1}\left(r+1, d, e+\chi\left(\mathcal{O}_{X}\right)\right)\right]=\sum_{i}[i]\left[\mathcal{M}(r, d, e)_{e+i}\right] .
$$

As observed in [KY00], this establishes a recursion

$$
\begin{aligned}
{\left[\mathcal{P}^{1}(r, d, e)\right] } & =\sum_{i}[e+i]\left[\mathcal{M}(r, d, e)_{e+i}\right]=[e][\mathcal{M}(r, d, e)]+\mathbb{L}^{e} \sum[i]\left[\mathcal{M}(r, d, e)_{e+i}\right] \\
& =[e][\mathcal{M}(r, d, e)]+\mathbb{L}^{e}\left[\mathcal{P}^{1}\left(r+1, d, e+\chi\left(\mathcal{O}_{X}\right)\right)\right]
\end{aligned}
$$

Because the dimension of $\mathcal{P}$ contains a term - re, iterating this leads to empty moduli spaces $\mathcal{P}$ when either (1) we are working on the a K3 surface where $e$ is increased by $\chi\left(\mathcal{O}_{X}\right)=2$ at each step, or (2) when $e>0$ and we are on the Abelian surface. (When $e=0$ on the Abelian surface, we learn that $\left[\mathcal{P}^{1}(r, d, 0)\right]$ is independent of $r \geqslant 0$, but we have not found any use for this fact.) In these cases we may sum the recursion (which is to say, the following sum is really a finite sum):

$$
\left[\mathcal{P}^{1}(r, d, e)\right]=\sum_{b=0}^{\infty}\left[e+b \chi\left(\mathcal{O}_{X}\right)\right] \mathbb{L}^{\sum_{j=0}^{b-1} e+j \chi\left(\mathcal{O}_{X}\right)}\left[\mathcal{M}\left(r+b, d, e+b \chi\left(\mathcal{O}_{X}\right)\right)\right]
$$

To evaluate this sum, it remains to (1) use the deformation equivalence of moduli of sheaves on K3 or Abelian surfaces and Hilbert schemes of points on these surfaces and then (2) plug in the formula for the Hodge polynomial of the Hilbert scheme [GS93]. For the K3 surfaces, this is done in [KY00]. We now proceed to the case of the Abelian surface, where one must moreover deal with the case where $e \leqslant 0$ in some other way.

\subsection{Abelian surfaces}

We change the notation slightly from the previous section, and write $\mathcal{M}_{\text {num }}(r, d, e)$ for what was $\mathcal{M}(r, d, e)$ : the moduli space of sheaves where $c_{1}=d$ is fixed only in cohomology. We now denote by $\mathcal{M}(r, C, e)$ the moduli space where $c_{1}=C$ is fixed in Pic, and similarly for the spaces $\mathcal{P}$. Note that the discussion there for $\mathcal{M}_{\text {num }}$ and $\mathcal{P}_{\text {num }}$ is equally valid for (what is here called) $\mathcal{M}$ and $\mathcal{P}$ and thus (3.1) holds for these spaces as well.

Let $A$ be an Abelian surface. Twisting by line bundles gives an isomorphism $A^{\vee} / A^{\vee}[r] \times$ 


\section{$\chi_{y}$-Genera of RELATive HiLbert schemes}

$\mathcal{M}(r, C, e) \cong \mathcal{M}_{\text {num }}(r, d, e)$. The space $\mathcal{M}_{\text {num }}(r, d, e)$ has dimension

$$
\operatorname{dim} \operatorname{Ext}^{1}(F, F)=2-\chi(F, F)=2-\int \operatorname{ch}(F)^{\vee} \operatorname{ch}(F)=2+C^{2}-2 r e=2 g(C)-2 r e .
$$

If this is greater than 2, then according to [Yos01, Theorem 0.1], the space $\mathcal{M}_{n u m}(r, d, e)$ is deformation equivalent to $A^{\vee} \times A^{[n]}$ for the appropriate $n$.

For $(g-1)>r e$, we have $\left[\mathcal{M}_{n u m}(r, d, e)\right]=\left[A^{\vee} \times A^{[g-1-r e]}\right]$ and $[\mathcal{M}(r, C, e)]=\left[A^{[g-1-r e]}\right]$. In particular, in this case $\chi_{-y}(\mathcal{M}(r, C, e))=0$. On the other hand, according to [Yos01, Lemma 4.19], when $r \mid(g-1)$, the space $\mathcal{M}(r, C,(g-1) / r)$ is a finite set of $r^{2}$ points. Thus for $e>0$, it follows from (3.1) that

$$
\left[\mathcal{P}^{1}(0, C, e)\right]=[e] \sum_{b=0}^{\infty} \mathbb{L}^{b e}[\mathcal{M}(b, C, e)]=[e]\left(\sum_{b=0}^{b<(g-1) / e} \mathbb{L}^{b e}\left[A^{[g-1-b e]}\right]+\mathbb{L}^{g-1} \sum_{b=(g-1) / e} b^{2}\right) .
$$

Now we treat the case of negative Euler characteristic. There are morphisms

$$
\begin{aligned}
& \pi_{+}: \mathcal{P}^{1}(0, C, e) \rightarrow \mathcal{M}(0, C, e), \quad(s, E) \mapsto E, \\
& \pi_{-}: \mathcal{P}^{1}(0, C,-e) \rightarrow \mathcal{M}(0, C, e), \quad(s, F) \mapsto \operatorname{Ext}_{\mathcal{O}_{S}}^{1}\left(F, \omega_{S}\right)=\operatorname{Hom}\left(F, \omega_{D}\right),
\end{aligned}
$$

where in the second line $D$ is the support curve of $F$. By Serre duality on the support curve we get $\left[\pi_{+}^{-1}(E)\right]-\mathbb{L}^{e}\left[\pi_{-}^{-1}(E)\right]=[e]$ for all $E \in \mathcal{M}(0, C, e)$. Furthermore, $\mathcal{M}(0, C, e)$ is stratified into locally closed subsets over which $\pi_{+}$and $\pi_{-}$are projectivizations of vector bundles. This gives

$$
\left[\mathcal{P}^{1}(0, C, e)\right]-\mathbb{L}^{e}\left[\mathcal{P}^{1}(0, C,-e)\right]=[e][\mathcal{M}(0, C, e)]=0 .
$$

We now pass from $[\cdot]$ to $\bar{\chi}_{-y}$. Note that in addition to specializing parameters, we must multiply by $y^{-\operatorname{dim} / 2}$. By the isomorphism with Hilbert schemes, we have $\operatorname{dim} \mathcal{P}^{1}(0, C, e)=$ $2(g-1)+e-1$. All terms containing $\left[A^{[\geqslant 1]}\right]$ vanish, leaving

$$
\begin{aligned}
\sum_{e \neq 0} t^{e} \bar{\chi}_{-y}\left(\mathcal{P}^{1}(0, C, e)\right) & =y^{1 / 2} \sum_{e \mid(g(C)-1)} \frac{y^{e}-1}{y-1}\left(y^{-e / 2} t^{e}+y^{e / 2} y^{-e} t^{-e}\right)\left(\frac{g-1}{e}\right)^{2} \\
& =\sum_{e \mid(g(C)-1)} \frac{y^{e / 2}-y^{-e / 2}}{y^{1 / 2}-y^{-1 / 2}}\left(t^{e}+t^{-e}\right)\left(\frac{g-1}{e}\right)^{2} .
\end{aligned}
$$

It remains to determine the contribution of sheaves with $e=0$. Let $g=g(C)$, and let

$$
f_{g}(y, t):=\sum_{e \in \mathbb{Z}} \bar{\chi}_{-y}\left(\mathcal{P}^{1}(0, C, e)\right) t^{e}=\sum_{e \in \mathbb{Z}} \bar{\chi}_{-y}\left(\mathcal{C}_{[A, C], g-2}^{[e+g-1]}\right) t^{e} .
$$

Thus by (1.2) the polynomials $N^{i}$ are defined by expanding

$$
f_{g}(y, t)=\sum_{i \geqslant 0} N_{[A, C], g-2}^{i}(y)\left(\frac{\left(1-y^{-1 / 2} t\right)\left(1-y^{1 / 2} t\right)}{t}\right)^{g-1-i} .
$$

We have shown in [GS14] that, for surfaces with trivial canonical bundle, $N_{[S, L], \delta}^{i}(y)=0$ for $i>\delta$. Applying this here, we see that $f_{g}(y, t)$ is a Laurent polynomial in $t$ and $y^{1 / 2}$, divisible by $\left(1-y^{-1 / 2} t\right)\left(1-y^{1 / 2} t\right) / t$. In particular, $f_{g}\left(y, y^{1 / 2}\right)=0$; in other words,

$$
\operatorname{Coeff}_{t^{0}}\left(f_{g}(y, t)\right)=-\sum_{e \mid(g(C)-1)} \frac{y^{e / 2}-y^{-e / 2}}{y^{1 / 2}-y^{-1 / 2}}\left(y^{e / 2}+y^{-e / 2}\right)\left(\frac{g-1}{e}\right)^{2},
$$


and

$$
f_{g}(y, t)=\sum_{e \mid(g(C)-1)} \frac{y^{e / 2}-y^{-e / 2}}{y^{1 / 2}-y^{-1 / 2}}\left(t^{e}+t^{-e}-y^{e / 2}-y^{-e / 2}\right)\left(\frac{g-1}{e}\right)^{2} .
$$

Putting $d:=e, n:=(g-1) / e$, we see that $\mathbb{A}=\sum_{g \geqslant 2} f_{g}(y, t) q^{g-1}$ is given by the second line of Theorem 1.5.

Remark 3.5. For the Hodge polynomial $\bar{h}(X)=(x y)^{-\operatorname{dim}(X) / 2} \sum_{p, q} \mathrm{~h}^{p, q}(X)(-x)^{p}(-y)^{q}$, the argument above gives

$$
\begin{aligned}
\sum_{e \neq 0} t^{e} \bar{h}\left(\mathcal{P}^{1}(0, C, e)\right)= & \sum_{e>0} \frac{(x y)^{e / 2}-(x y)^{-e / 2}}{(x y)^{1 / 2}-(x y)^{-1 / 2}} \times \\
& \times\left(\bar{h}\left(A^{[g-1]}\right) t^{e}+\left(t^{e}+t^{-e}\right)\left(\sum_{0<b<(g-1) / e} \bar{h}\left(A^{[g-1-b e]}\right)+\sum_{b=(g-1) / e} b^{2}\right)\right) .
\end{aligned}
$$

\section{The refined invariants for surfaces with $K_{S}$ numerically trivial}

In this section we prove Corollary 1.9 and Theorem 1.10. Let

$$
x:=t+t^{-1}-y^{1 / 2}-y^{-1 / 2}=\frac{1}{t}\left(1-t y^{1 / 2}\right)\left(1-t y^{-1 / 2}\right)=-y^{-1 / 2}\left(1-y^{1 / 2} t\right)\left(1-y^{1 / 2} / t\right) .
$$

By Theorem 1.2 it is enough to prove Corollary 1.9 for K3 surfaces and Abelian surfaces. Let $S$ be a K3 surface or an Abelian surface with $\operatorname{Pic}(S)=\mathbb{Z}$ generated by a line bundle $L_{g}$ with $g\left(L_{g}\right)=g$. These exist for any $g \geqslant 2$ : for Abelian surfaces we can take a principally polarized Abelian surface with a polarization of type $(1, g-1)$. For K3 surfaces this result can for instance be found in [Che04, Proposition 1.12], where the surfaces are defined as deformations of embeddings in $\mathbb{P}^{g}$ of hypersurfaces in $\mathbb{P}^{1} \times \mathbb{P}^{2}$ and in $\mathbb{P}^{2}$-bundles over $\mathbb{P}^{1}$.

We know

$$
\sum_{i} N_{\left[S, L_{g}\right], \chi\left(L_{g}\right)-1-k}^{i}(y) x^{g-i-1}=\operatorname{Coeff}_{q^{g-1}}\left[\mathbb{X}^{k} \mathbb{K}^{\chi\left(\mathcal{O}_{S}\right) / 2} \mathbb{A}^{1-\chi\left(\mathcal{O}_{S}\right) / 2}\right] .
$$

We know $N_{\left[S, L_{g}\right], \chi\left(L_{g}\right)-1-k}^{i}=0$ for $i>\chi\left(L_{g}\right)-1-k=g-\left(k+2-\chi\left(\mathcal{O}_{S}\right)\right)$.

This means

$$
\mathbb{X}^{k} \mathbb{K}^{\chi\left(\mathcal{O}_{S}\right) / 2} \mathbb{A}^{1-\chi\left(\mathcal{O}_{S}\right) / 2} \in x^{k+1-\chi\left(\mathcal{O}_{S}\right)} \mathbb{Q}[x]\left[q^{-1}\right][[q]]
$$

and

$$
\sum_{g} N_{\left[S, L_{g}\right], \chi\left(L_{g}\right)-1-k}^{\chi\left(L_{g}\right)-1-k}(y) q^{g-1}=\left.\frac{\mathbb{X}^{k} \mathbb{K}^{\chi\left(\mathcal{O}_{S}\right) / 2} \mathbb{A}^{1-\chi\left(\mathcal{O}_{S}\right) / 2}}{x^{k+1-\chi\left(\mathcal{O}_{S}\right)}}\right|_{t=y^{1 / 2}} .
$$

By Theorem 1.5 and the fact that $\left.[d]_{y}\right|_{y=1}=d$, we get

$$
\left.\frac{\mathbb{A}}{x}\right|_{t=y^{1 / 2}}=\sum_{n>0, d>0} n^{2} d[d]_{y}^{2} q^{n d}=D \widetilde{D G}(y, q) .
$$

As $\mathbb{H}=D^{-1} \mathbb{A}$, we also see

$$
\left.\frac{\mathbb{H}}{x}\right|_{t=y^{1 / 2}}=\widetilde{D G}_{2}(y, q)
$$




\section{$\chi_{y}$-GeneRA OF RELATIVE HiLbert SCHEMES}

By Theorem 1.6, we get $\left.(x \mathbb{K})\right|_{t=y^{1 / 2}}=\widetilde{\Delta}(y, q)$. As $\mathbb{X} \in \mathbb{H} \cdot(1+\mathbb{Q}[y][[\mathbb{H}]])$, we find

$$
\left.\frac{\mathbb{X}}{x}\right|_{t=y^{1 / 2}}=\left.\frac{\mathbb{H}}{x}\right|_{t=y^{1 / 2}}=\widetilde{D G}_{2}(y, q)
$$

Substituting, we have proven Corollary 1.9:

$$
\sum_{g} N_{\left[S, L_{g}\right], \chi\left(L_{g}\right)-1-k}^{\chi\left(L_{g}\right)-1-k}(y) q^{g-1}=\frac{\widetilde{D G}_{2}(y, q)^{k}\left(D \widetilde{D G}_{2}(y, q)\right)^{1-\chi\left(\mathcal{O}_{S}\right) / 2}}{\Delta(y, q)^{\chi\left(\mathcal{O}_{S}\right) / 2}} .
$$

Now we prove Theorem 1.10. This proof is due to Zagier (except for the easy deduction of (4.2)). As before let $e^{z}:=y, e^{z_{1}}:=y_{1}:=t y^{1 / 2}, e^{z_{2}}:=y_{2}=y^{1 / 2} / t$. Note that $y_{1} y_{2}=y$. We can rewrite the formula of Theorem 1.6 as follows:

$$
\mathbb{K}=\frac{y^{-1 / 2}-y^{1 / 2}}{\Delta(q)} \frac{\theta^{\prime}(0)^{3}}{\theta\left(y_{1}\right) \theta\left(y_{2}\right) \theta(y)}=\frac{1}{\left(y^{-1 / 2}-y^{1 / 2}\right) \widetilde{\Delta}(y, q)} \frac{\theta^{\prime}(0) \theta\left(y_{1} y_{2}\right)}{\theta\left(y_{1}\right) \theta\left(y_{2}\right)} .
$$

Let $\varepsilon:=\left(y_{1}-1\right)\left(y_{2}-1\right) /(y-1)=x /\left(y^{-1 / 2}-y^{1 / 2}\right)$. Then by (1.1) we have

$$
x \widetilde{\Delta}(y, q) \mathbb{K}=1-\varepsilon \sum_{n d>0} \operatorname{sgn}(d) y_{2}^{n-d} y^{d} q^{n d}=1-\varepsilon \sum_{n d>0} \operatorname{sgn}(d) e^{-(d-n) z_{2}} y^{d} q^{n d} .
$$

Note that $\varepsilon=\varepsilon\left(z_{2}\right)=\frac{1}{y-1}\left(y e^{-z_{2}}-1\right)\left(e^{z_{2}}-1\right)$ is a power series in $\mathbb{Q}[1 /(y-1)]\left[\left[z_{2}\right]\right]$, starting with $z_{2}$. Let $z_{2}(\varepsilon)$ be the inverse series. Then the formula for $\mathbb{K}$ of the theorem follows from (4.1) together with the claim that

$$
z_{2}(\varepsilon)=\sum_{n \geqslant 0} \frac{s_{n}(y)}{\left(y^{1 / 2}-y^{-1 / 2}\right)^{n}} \frac{\varepsilon^{n+1}}{n+1}=-S(y, x) .
$$

By the Lagrange inversion formula $\operatorname{Coeff}_{\varepsilon^{n}} z_{2}=(1 / n) \operatorname{Res}_{z_{2}=0} \varepsilon^{-n}$, we need to see that

$$
(y-1)^{n} \operatorname{Res}_{z_{2}=0} \varepsilon^{-n-1}=\operatorname{Coeff}_{x^{n}}((1+x)(1+x y))^{n} .
$$

We put $Y:=(y-1)$ and $T=e^{z_{2}}-1$; then

$$
(y-1)^{n} \operatorname{Res}_{z_{2}=0} \varepsilon^{-n-1}=\operatorname{Coeff}_{T^{n}} \frac{Y^{2 n+1}(1+T)^{n}}{(Y-T)^{n+1}}=\operatorname{Coeff}_{T^{n}} \frac{(1+T Y)^{n}}{(1-T)^{n+1}}=\sum_{l=0}^{n}\left(\begin{array}{c}
n \\
l
\end{array}\right)\left(\begin{array}{c}
2 n-l \\
n
\end{array}\right) Y^{l} .
$$

On the other hand,

$$
\operatorname{Coeff}_{x^{n} Y^{l}}((1+x)(1+x(1+Y)))^{n}=\sum_{k=0}^{n}\left(\begin{array}{l}
n \\
k
\end{array}\right)^{2}\left(\begin{array}{l}
k \\
l
\end{array}\right)=\sum_{k=0}^{n}\left(\begin{array}{l}
n \\
k
\end{array}\right)\left(\begin{array}{l}
n-l \\
n-k
\end{array}\right)\left(\begin{array}{l}
n \\
l
\end{array}\right)=\left(\begin{array}{c}
2 n-l \\
n
\end{array}\right)\left(\begin{array}{l}
n \\
l
\end{array}\right) .
$$

This establishes (4.2) and thus the formula for $\mathbb{K}$. To prove the formula for $\mathbb{A}$, note that by the definition $A(y, q)=\sum_{n d>0} \operatorname{sgn}(d) n^{2}\left(y^{d}-1\right) q^{n d}$. Thus, by Theorem 1.5, we have

$$
\begin{aligned}
\left(y^{1 / 2}-y^{-1 / 2}\right) \mathbb{A} & =A\left(y_{1}, q\right)+A\left(y_{2}, q\right)-A(y, q)=\sum_{n d>0} \operatorname{sgn}(d) n^{2}\left(y_{1}^{d}+y_{2}^{d}-\left(y^{d}-1\right)\right) q^{n d} \\
& =\sum_{n d>0} \operatorname{sgn}(d) n^{2}\left(y_{1}^{d}-y_{2}^{-d}-\left(y^{d}-1\right)\right) q^{n d}=\sum_{n d>0} \operatorname{sgn}(d) n^{2}\left(e^{-z_{2} d}-1\right)\left(y^{d}-1\right) q^{n d}
\end{aligned}
$$

and we can use (4.2) again. This finishes the proof of the formula for $\mathbb{A}$. The formula for $\mathbb{H}$ now follows directly from the definition $\mathbb{H}=D^{-1} \mathbb{A}$. 


\section{Lothar Göttsche And Vivek Shende}

\section{ACKNOWLEDGEMENTS}

We thank Don Zagier for the contributions mentioned in the last paragraph of the introduction, and Kōta Yoshioka for helpful correspondence about sheaves on Abelian surfaces. Part of this work was carried out while the first-named author was at the Max-Planck-Institut für Mathematik, Bonn.

\section{REFERENCES}

BG14 F. Block and L. Göttsche, Refined curve counting with tropical geometry, Compos. Math. (to appear), arXiv:1407.2901.

BL00 J. Bryan and N.C. Leung, The enumerative geometry of K3 surfaces and modular forms, J. Amer. Math. Soc. 13 (2000), no. 2, 371-410; http://dx.doi.org/10.1090/ S0894-0347-00-00326-X.

Che04 X. Chen, Rational curves on K3 surfaces, available at http://www.cms.zju.edu.cn/ UploadFiles/AttachFiles/2004723191336353.pdf.

EGL01 G. Ellingsrud, L. Göttsche, and M. Lehn, On the cobordism class of the Hilbert scheme of a surface, J. Algebraic Geom. 10 (2001), no. 1, 81-100.

Göt98 L. Göttsche, A conjectural generating function for numbers of curves on surfaces, Comm. Math. Phys. 196 (1998), no. 3, 523-533; http://dx.doi.org/10.1007/s002200050434.

GS93 L. Göttsche and W. Soergel, Perverse sheaves and the cohomology of Hilbert schemes of smooth algebraic surfaces, Math. Ann. 296 (1993), no. 2, 235-245; http://dx.doi.org/10.1007/ BF01445104.

GS14 L. Göttsche and V. Shende, Refined curve counting on complex surfaces, Geom. Topol. 18 (2014), no. 4, 2245-2307. http://dx.doi.org/10.2140/gt.2014.18.2245

GZ98 L. Göttsche and D. Zagier, Jacobi forms and the structure of Donaldson invariants for 4manifolds with $b_{+}=1$, Selecta Math. (N.S.) 4 (1998), no. 1, 69-115; http://dx.doi.org/10. $1007 / \mathrm{s} 000290050025$.

Hir95 F. Hirzebruch, Topological methods in algebraic geometry, Classics Math. (Springer-Verlag, Berlin, 1995); http://dx.doi.org/10.1007/978-3-642-62018-8.

IM13 I. Itenberg and G. Mikhalkin, On Block-Göttsche multiplicities for planar tropical curves, Int. Math. Res. Not. IMRN 2013 (2013), no. 23, 5289-5320; http://dx.doi.org/10.1093/imrn/ rns207.

KS13 S. L. Kleiman and V.V. Shende, On the Göttsche threshold, with an appendix by Ilya Tyomkin, in A Celebration of Algebraic Geometry, Clay Math. Proc., vol. 18 (Amer. Math. Soc., Providence, RI, 2013), 429-449.

KST11 M. Kool, V. Shende, and R. P. Thomas, A short proof of the Göttsche conjecture, Geom. Topol. 15 (2011), no. 1, 397-406; http://dx.doi.org/10.2140/gt.2011.15.397.

KT14a M. Kool and R. Thomas, Reduced classes and curve counting on surfaces I: theory, Algebr. Geom. 1 (2014), no. 3, 334-383; http://dx.doi.org/10.14231/AG-2014-017

KT14b_ Reduced classes and curve counting on surfaces II: calculations, Algebr. Geom. 1 (2014), no. 3, 384-399. http://dx.doi.org/10.14231/AG-2014-018.

KY00 T. Kawai and K. Yoshioka, String partition functions and infinite products, Adv. Theor. Math. Phys. 4 (2000), no. 2, 397-485.

LP95 J. Le Potier, Faisceaux semi-stables et systèmes cohérents, Vector Bundles in Algebraic Geometry (Durham, 1993), London Math. Soc. Lecture Note Ser., vol. 208 (Cambridge Univ. Press, Cambridge, 1995), 179-239.

Mik05 G. Mikhalkin, Enumerative tropical algebraic geometry in $\mathbb{R}^{2}$, J. Amer. Math. Soc. 18 (2005), no. 2, 313-377; http://dx.doi.org/10.1090/S0894-0347-05-00477-7. 


\section{$\chi_{y}$-Genera of RElative Hilbert SCHEMES}

MNOP06 D. Maulik, N. Nekrasov, A. Okounkov, and R. Pandharipande, Gromov-Witten theory and Donaldson-Thomas theory. I, Compos. Math. 142 (2006), no. 5, 1263-1285; http://dx.doi. org/10.1112/S0010437X06002302.

MPT10 D. Maulik, R. Pandharipande, and R.P. Thomas, Curves on K3 surfaces and modular forms, with an appendix by a. pixton, J. Topol. 3 (2010), no. 4, 937-996; http://dx.doi.org/10. 1112/jtopol/jtq030.

PT09 R. Pandharipande and R.P. Thomas, Curve counting via stable pairs in the derived category, Invent. Math. 178 (2009), no. 2, 407-447; http://dx.doi.org/10.1007/s00222-009-0203-9.

PT10 Stable pairs and BPS invariants, J. Amer. Math. Soc. 23 (2010), no. 1, 267-297; http://dx.doi.org/10.1090/S0894-0347-09-00646-8.

Tze12 Y.-j. Tzeng, A proof of the Göttsche-Yau-Zaslow formula, J. Differential Geom. 90 (2012), no. 3, 439-472; http://projecteuclid.org/euclid.jdg/1335273391.

Yos99 K. Yoshioka, Some examples of Mukai's reflections on K3 surfaces, J. Reine Angew. Math. 515 (1999), 97-123; http://dx.doi.org/10.1515/crll.1999.080.

Yos01_ Moduli spaces of stable sheaves on abelian surfaces, Math. Ann. 321 (2001), no. 4, 817-884; http://dx.doi.org/10.1007/s002080100255.

Zag91 D. Zagier, Periods of modular forms and Jacobi theta functions, Invent. Math. 104 (1991), no. 3, 449-465; http://dx.doi.org/10.1007/BF01245085.

Lothar Göttsche gottsche@ictp.it

International Centre for Theoretical Physics, Strada Costiera 11, I-34151 Trieste, Italy

Vivek Shende vivek@math.berkeley.edu

Department of Mathematics, University of California, Berkeley, 970 Evans Hall, Berkeley, CA 94720-3840, USA 